\title{
Usefulness of ultrasonography and biochemical features in the diagnosis of cholestatic jaundice in infants
}

\author{
M S Choopa, ${ }^{1} \mathrm{MB}$ ChB, FC Paed (SA); C Kock, ${ }^{1} \mathrm{MB}$ ChB, FC Paed (SA), Cert Gastroenterology Paed (SA); \\ S O M Manda, ${ }^{2} \mathrm{PhD}$ (Stats); A J Terblanche, ${ }^{1} \mathrm{MB} \mathrm{ChB}$, FC Paed (SA), Dip Allerg (SA), Cert Gastroenterology Paed (SA); \\ D F Wittenberg, ${ }^{1} \mathrm{MD}$, FCP (Paed) (SA)

\begin{abstract}
${ }^{1}$ Division of Gastroenterology, Department of Paediatrics and Child Health, School of Medicine, Faculty of Health Sciences, University of Pretoria, South Africa

${ }^{2}$ Biostatistics Research Unit, South African Medical Research Council, Pretoria, South Africa, and School of Mathematics, Statistics and Computer Science, University of KwaZulu-Natal, Pietermaritzburg, South Africa
\end{abstract}

Corresponding author: M S Choopa (mchoopa@yahoo.co.uk)

This work was a Master of Medicine in Paediatrics research study.

\begin{abstract}
Background. Biliary atresia is a common cause of cholestasis. In our experience, patients with biliary atresia are referred late, with the diagnosis based on an absent gall bladder at ultrasonography. Such late referrals may render patients inoperable and not acceptable for formal intraoperative diagnosis.

Objectives. To determine the usefulness of an absent gall bladder on ultrasonography, and of biochemical features, in differentiating biliary atresia from other causes of cholestasis, using liver needle biopsy as a gold standard.

Methods. A retrospective file review of 150 infants presenting with cholestasis to Steve Biko Academic Hospital Paediatric Gastroenterology and Hepatology Unit from January 2008 to August 2014 was undertaken. Clinical, serum biochemical, abdominal ultrasonography and liver histology findings were analysed. Three groups were compared, based on liver histology findings, consisting of patients with biliary atresia, neonatal hepatitis, and other diagnoses, respectively.

Results. A total of 66/150 patients had biliary atresia, based on liver histology findings of extrahepatic obstruction. Their mean age was 4.7 (2.9) months, higher than in the other groups. In those with biliary atresia, the age at diagnosis, splenomegaly, and gamma glutamyl transferase (GGT), aspartate transaminase (AST) and GGT/AST ratio values were significantly different from the other groups. Total and conjugated bilirubin levels were similar among the groups. Ultrasonography was $69.7 \%$ sensitive, $98.8 \%$ specific and had a positive predictive value of $97.9 \%$ for biliary atresia. Ultrasonography missed $30.3 \%$ of patients with biliary atresia.

Conclusion. Ultrasonography has poor sensitivity but good specificity in screening for biliary atresia; however, other investigations are necessary to confirm the diagnosis.
\end{abstract}

S Afr J Child Health 2016;10(1):75-78. DOI:10.7196/SAJCH.2016.v10i1.1075

\begin{abstract}
Cholestasis results from reduced excretion of bile due to a number of disorders $^{[1]}$ and is defined as conjugated bilirubin more than $20 \%$ of the total bilirubin when the total bilirubin is $85 \mu \mathrm{mol} / \mathrm{L}$ or more, and $17 \mu \mathrm{mol} / \mathrm{L}$ or more when the total bilirubin is $85 \mu \mathrm{mol} / \mathrm{L}$ or less. ${ }^{[2]}$ It is good clinical practice to determine the conjugated fraction of bilirubin in any infant who remains jaundiced beyond the age of 2 weeks $^{[3,4]}$ and ultrasonography is one of the first investigations that is performed ${ }^{[2]}$ Identification of a normal gall bladder on sonogram is highly predictive of the absence of biliary atresia. ${ }^{[5]}$ Visualisation of the gall bladder may be operator dependent and is also influenced by the patient feeding or fasting, even though biliary atresia can be accurately diagnosed when performed by an experienced sonographer. ${ }^{[6]}$ The presence or absence of the triangular cord sign (TCS) on ultrasonography of the gall bladder has been described to assist in the diagnosis of biliary atresia and is the visualisation of a triangular or tubular echogenic density cranial to the portal vein bifurcation on a transverse or longitudinal ultrasonograhic scan. ${ }^{[7]}$ After finding a TCS, explorative laparotomy should be done to confirm biliary atresia, while patients in whom the TCS is absent should have hepatobiliary scintigraphy. ${ }^{[7]}$

Cholestasis can also be assessed by using serum biochemical markers such as liver function tests (total and conjugated bilirubin) and liver enzymes (alanine transaminase (ALT), aspartate
\end{abstract}

transaminase (AST), alkaline phosphatase (ALP) and gamma glutamyl transferase (GGT)). The serum GGT is usually higher in biliary atresia than in other causes of neonatal cholestasis when correlated with age. ${ }^{[8]}$ Liver biopsy shows extrahepatic biliary obstruction by varying degrees of portal tract fibrosis, oedema, ductular proliferation and cholestasis with appearance of bile plugs. ${ }^{[8]}$ It has been described as the most accurate investigation (99-100\%) in differentiating biliary atresia from other causes of cholestasis. ${ }^{[2,4]}$

Idiopathic neonatal hepatitis and biliary atresia are common and make up $70-80 \%$ of causes of cholestasis in term infants. ${ }^{[1,9,10]}$ The incidence of biliary atresia ranges from 1 in 5000 in Taiwan to 1 in $17000-19000$ live births in the UK and France. ${ }^{[8]}$

Biliary atresia is characterised by progressive obliterative cholangiopathy ${ }^{[11]}$ Therefore, it is crucial to diagnose biliary atresia for early surgical intervention ${ }^{[3]}$ using the Kasai procedure, which is the resection of all the extrahepatic bile ducts and anastomosis of a jejunal en Y loop at the porta hepatis. ${ }^{[1]}$ This procedure improves outcome and long-term survival of the infant's native liver when performed between the ages of 45 and 60 days. ${ }^{[3,4]}$ Liver transplantation is the only alternative therapeutic procedure ${ }^{[12]}$ in those for whom the Kasai procedure is not feasible or has failed.

In view of our clinical experience that many patients are only referred for evaluation of cholestasis after the finding of an absent 
gall bladder on ultrasonography examination, we aimed to determine the accuracy of ultrasonography in the diagnosis of biliary atresia and to determine the differences in clinical, biochemical and histological findings in infants who presented with different forms of cholestasis.

\section{Methods \\ Data}

A retrospective review of files of patients aged $0-12$ months, who presented with cholestasis to the Paediatric Gastroenterology and Hepatology Unit, Steve Biko Academic Hospital, Pretoria from January 2008 to August 2014, was undertaken. Ethical approval (Protocol number: 33/2015) was obtained from the University of Pretoria Health Sciences Research Ethics Committee. Permission to review the hospital files was obtained from the Chief Executive Officer of the hospital. Out of 199 patient files reviewed, 150 patients were included in the study. Forty-nine patients had neither ultrasound of liver, liver biopsy nor both, or were above 1 year of age.

The following details were extracted from the files: gender, age at diagnosis, biochemical results of liver enzymes (ALT, AST, GGT, ALP) and liver function (total and conjugated bilirubin) at first presentation, ultrasonography reports and histological report of liver biopsies.

Patients aged 0 - 12 months who presented with cholestasis, and had either liver ultrasonography or liver biopsy or both, were included. Patients aged $>12$ months, or who did not have cholestasis, or who did not have an ultrasound or biopsy of the liver, were excluded. Biliary atresia was defined as the presence of extrahepatic obstruction on liver biopsy, based on bile duct proliferation, portal fibrosis and canalicular bile stasis.

We used liver histology findings even though this is an imperfect gold standard, because the majority of our patients were referred too late to be deemed operable for an intraoperative diagnosis during laparotomy. Only 12 of the 66 patients with histological evidence of extrahepatic obstruction had exploratory laparotomies, and none had cholangiograms.

Hepatomegaly was defined as a liver that was palpable $\geq 2 \mathrm{~cm}$ below the right costal margin in the midclavicular line and splenomegaly was defined as any-sized spleen palpable below the left costal margin. The TCS was infrequently reported on, therefore we included patients with absence of a gall bladder on ultrasonography as possibly having biliary atresia.

The liver enzyme, total and conjugated bilirubin, and histology results of the liver biopsies and abdominal ultrasonography, were tabulated.

\section{Statistical methods}

Continuous data were expressed as means with standard deviations (SDs) and categorical data were summarised using frequencies and percentages. The independent $t$-test was used for comparison of normally distributed data; otherwise non-parametric alternatives were used. Performance of the diagnostic methods was evaluated using the area under the receiver-operating curve (ROC), known as the C-index. We used the kappa ( $\kappa$ ) statistic to assess the proportion of observed-to-expected agreement between ultrasonography findings and liver enzymes with liver histology. Stata 13 (StataCorp, Texas, USA) was used for data analysis and $p<0.05$ was considered to be statistically significant.

\section{Results}

The liver histology findings were divided into three groups: group 1 consisted of those with the features of biliary atresia, group 2 had the features of neonatal hepatitis due to sepsis or cytomegalovirus infection, and group 3 consisted of other diagnoses. The last group included intrahepatic paucity of bile ducts, Caroli disease, veno-occlusive disease, alpha-1 antitrypsin deficiency and steatosis. The distribution of clinical and serum biochemical findings across the groups is shown (Table 1).

The overall mean (SD) age at diagnosis of the 150 patients was 3.7 (2.85) months, and differed significantly across the groups, with the biliary atresia group having the highest age of 4.7 (2.9) months $(p<0.05)$ at diagnosis. There were 89 boys and 61 girls of different races, with no significant difference between the sexes in those with biliary atresia.

There were significant differences in the proportions of patients with splenomegaly in the three groups, with more patients with biliary atresia presenting with splenomegaly compared with the other two groups. The GGT, AST and their ratio had different distributions across the groups, with GGT and the ratio to AST being higher in patients with biliary atresia $(p<0.05)$. AST was significantly higher in the neonatal hepatitis group than in the other two groups $(p<0.05)$. Of the biochemical features in the

Table 1. Clinical, ultrasonography and biochemical parameters by cause of cholestasis

\begin{tabular}{|c|c|c|c|c|}
\hline & $\begin{array}{l}\text { Biliary atresia } \\
(N=66,44 \%)\end{array}$ & $\begin{array}{l}\text { Neonatal hepatitis } \\
(N=37,24.7 \%)\end{array}$ & $\begin{array}{l}\text { Other diagnoses } \\
(N=47,31.3 \%)\end{array}$ & $p$-value \\
\hline $\begin{array}{l}\text { Age (months), } \\
\text { mean (SD) }\end{array}$ & $4.7(2.9)$ & $2(1.74)$ & $3.6(2.7)$ & 0.000 \\
\hline \multicolumn{5}{|l|}{ Gender, $n(\%)$} \\
\hline Male & $32(48.5)$ & $26(70.3)$ & $31(66.0)$ & 0.052 \\
\hline Female & $34(51.5)$ & $11(29.7)$ & $16(34.0)$ & 0.052 \\
\hline Hepatomegaly, $n$ (\%) & $48(72.0)$ & $29(80.6)$ & $32(68.1)$ & 0.444 \\
\hline Splenomegaly, $n(\%)$ & $44(66.7)$ & $14(37.8)$ & $30(63.8)$ & 0.018 \\
\hline \multicolumn{5}{|l|}{ GGT (IU/L) } \\
\hline Mean (SD) & $604.5(717.1)$ & $197.1(232.9)$ & $408.3(491)$ & 0.0026 \\
\hline Median (IQR) & $382.5(139-714)$ & $114(85-212)$ & $159(68-688)$ & \\
\hline \multicolumn{5}{|l|}{ AST (IU/L) } \\
\hline Mean (SD) & $277.8(184.7)$ & $428.6(396.4)$ & $269.9(199.5)$ & 0.0082 \\
\hline Median (IQR) & $219(142-374)$ & $323(181-478)$ & $213(140-326)$ & \\
\hline \multicolumn{5}{|l|}{ GGT/AST ratio } \\
\hline Mean (SD) & $2.9(3.7)$ & $1.1(1.8)$ & $2.4(3.93)$ & 0.039 \\
\hline Median (IQR) & $1.37(0.53-3.58)$ & $0.35(0.18-1.13)$ & $0.97(0.3-3.2)$ & \\
\hline \multicolumn{5}{|l|}{ Total bilirubin (IU/L) } \\
\hline Mean (SD) & $219.6(76.8)$ & $231.3(115.8)$ & $241.9(120.7)$ & 0.5179 \\
\hline Median (IQR) & $211.5(160-261)$ & $218(160-274)$ & $210(151-319)$ & \\
\hline \multicolumn{5}{|l|}{$\begin{array}{l}\text { Conjugated bilirubin } \\
\text { (IU/L) }\end{array}$} \\
\hline Mean (SD) & $133.3(55.5)$ & $131.9(79.9)$ & $141.9(76.7)$ & 0.7569 \\
\hline Median (IQR) & $125(97-151)$ & $109(71-161.5)$ & $115(92-164)$ & \\
\hline $\begin{array}{l}\text { Gall bladder visible on } \\
\text { ultrasound, } n(\%)\end{array}$ & $20 / 66(30.3)$ & $37 / 37(100)$ & 46/47 (97.9) & 0.000 \\
\hline
\end{tabular}




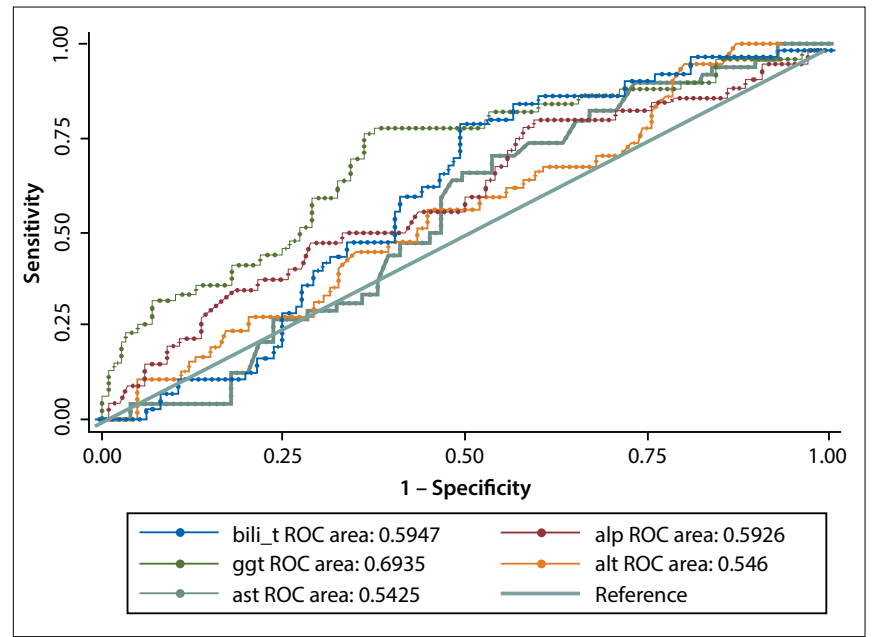

Fig. 1. ROC with area under the curve (AUC) of liver enzymes. The C-index of GGT was 0.67 (95\% CI 0.58 - 0.76) while the C-indices of AST, ALT and ALP with 95\% CI were 0.47, 0.52 and 0.58, respectively.

Table 2. ROC curve and AUC data to determine diagnostic accuracy of biliary atresia using serum biochemical investigations

\begin{tabular}{lccll}
\hline & Obs, $\boldsymbol{n}$ & Area & SE & 95\% CI \\
\hline ALP & 149 & 0.585 & 0.047 & $0.492-0.678$ \\
GGT & 149 & 0.671 & 0.045 & $0.582-0.759$ \\
ALT & 149 & 0.521 & 0.048 & $0.428-0.615$ \\
AST & 149 & 0.468 & 0.048 & $0.375-0.562$ \\
GGT to AST ratio & 150 & 0.652 & 0.045 & $0.564-0.739$ \\
Obs = observations; SE = standard error. & & &
\end{tabular}

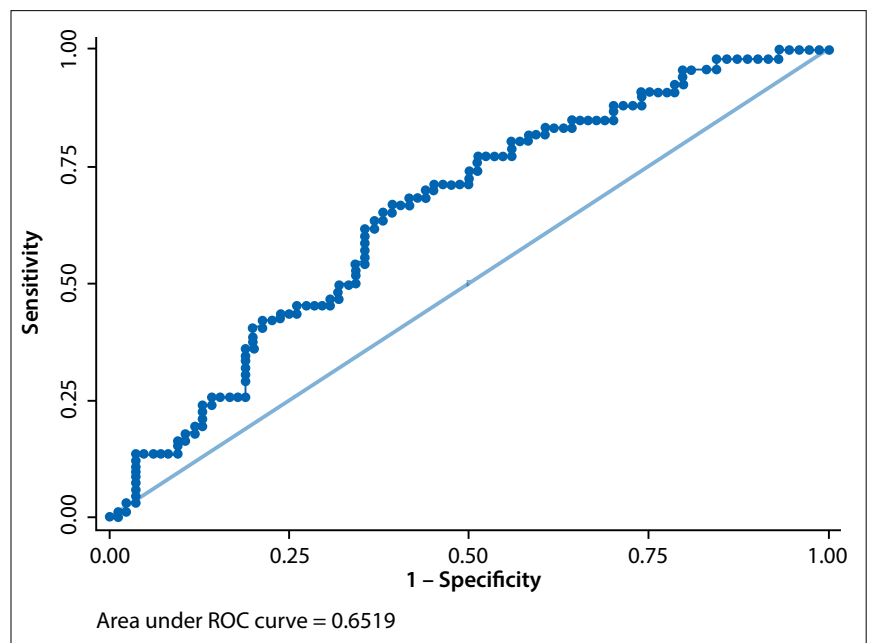

Fig. 2. GGT to AST ratios shown as ROC and AUC. Graph indicates the AUR and ROC of GGT/AST ratio performance in the diagnosis of biliary atresia. The $C$-index of the GGT/AST ratio was 0.65 (95\% CI 0.56 - 0.73).

biliary atresia group, the C-indices of GGT (Fig. 1, Table 2), and of the GGT to AST ratio (Fig. 2, Table 2), were statistically significant because their $95 \%$ confidence intervals did not include $0.5 .^{[13]}$ The total and conjugated bilirubin were similarly raised across all groups.

The performance of the ultrasonography finding of an absent gall bladder in determining biliary atresia in infants with cholestasis is outlined (Table 3 ). The sensitivity of ultrasonography was $69.7 \%$,
Table 3. Ultrasonography performance in determining biliary atresia in infants with cholestasis (\%)

\begin{tabular}{ll}
\hline Sensitivity & 69.7 \\
Specificity & 98.8 \\
Positive predictive value & 97.9 \\
Negative predictive value & 80.5
\end{tabular}

specificity was $98.8 \%$, and positive predictive value was $97.9 \%$. Thus, in our series, ultrasonography was only able to identify biliary atresia in $69.7 \%$ of the 66 patients who had features of biliary atresia on histology. Of the 84 patients who did not have features of biliary atresia on histology, $98 \%$ had gall bladders on ultrasonography examination. The accuracy of the ultrasonography was therefore determined to be between 70 and $99 \%$, and the agreement, measured by $\kappa$, was $0.71(p<0.05)$.

\section{Discussion}

Of the 150 infants reviewed, 66 (44\%) had biliary atresia (extrahepatic biliary obstruction on liver histology). Clinical, serum biochemistry and ultrasonography parameters were compared against liver histology results to assess their usefulness in the diagnosis of biliary atresia in infants with cholestasis. The mean age at diagnosis of biliary atresia was 4.7 (2.9) months and was higher than that of the other groups. Many patients with cholestasis were referred for further management of suspected biliary atresia after initial ultrasonography had indicated the presence of a gall bladder. In most of these patients, a diagnosis of biliary atresia was made. In our study, $30.3 \%$ of patients with extrahepatic biliary obstruction on histology were diagnosed as having gall bladders on ultrasonography examination. Many patients presented in advanced liver disease with liver dysfunction and portal hypertension, and the Kasai procedure would not have improved their outcome, which is improved if surgery is performed before the age of 8 weeks. ${ }^{[3]}$ Other factors that may have contributed to the late presentation of patients with biliary atresia include failure of clinicians to realise the importance of persisting cholestasis and failure to detect jaundice clinically in dark-skinned babies.

There was no significant difference in the size of the liver among the groups and more than $70 \%$ of all patients had hepatomegaly. Splenomegaly was more frequent in those with biliary atresia due to the late presentation of patients, indicating portal hypertension. ${ }^{[8]}$ One of the reasons fewer patients with neonatal hepatitis had splenomegaly may have been due to the shorter duration of the disease. One study showed hepatomegaly and splenomegaly in $50 \%$ of cases of cholestasis in infants; ${ }^{[14]}$ however, in our study, $73 \%$ of infants with cholestasis had hepatomegaly while $59 \%$ had splenomegaly.

Park et al. ${ }^{[7]}$ described the use of TCS in the diagnosis of biliary atresia, the finding of which was followed by mandatory explorative laparotomy. The features of TCS are a triangular or tubular shaped echogenic density cranial to the portal vein bifurcation on ultrasonography of the gall bladder. ${ }^{[7]}$ In our study, this sign was reported on very infrequently, therefore we assessed the value of visualising the gall bladder in the diagnosis of biliary atresia. Ultrasonography had a sensitivity of $69.7 \%$, a specificity of $98.8 \%$ and an accuracy of $86.0 \%$ in the diagnosis of biliary atresia. A total of 20 of the 103 patients who had gall bladders on ultrasonography had extrahepatic biliary obstruction on histology. Therefore, ultrasonography did not identify $30.3 \%$ of the 66 cases with biliary atresia. Ultrasonography was only able to exclude biliary atresia correctly in $80.5 \%$ of the 103 patients with a visible gall bladder (negative predictive value). These findings were marginally comparable to those of Park et al. ${ }^{[7]}$ who reported that the use of 
ultrasonography in the diagnosis of biliary atresia using the TCS had a sensitivity, specificity and accuracy of $85 \%, 100 \%$ and $95 \%$, respectively. Dehghani et al. ${ }^{[9]}$ reported a sensitivity of $52 \%$ and specificity of $76.1 \%$ using ultrasonography in the diagnosis of biliary atresia. However, experience in performing ultrasonography is important. ${ }^{[6]}$

Serum GGT levels were significantly higher in infants with biliary atresia (median 382.5, IQR 139 - 714) than with neonatal hepatitis (median 114, IQR 85 - 212) or with other diagnoses (median 159, IQR 68 - 688), $p<0.05$. GGT is usually higher in biliary atresia than in other causes of neonatal cholestasis, especially when correlated with age. ${ }^{[8]}$ The GGT levels in our series were similar to those reported by Tang et al. ${ }^{[15]}$ which were significantly higher in patients with biliary atresia than in neonatal hepatitis, and with a GGT/AST ratio $>2$ in 55/68 infants with biliary atresia and 15/54 with neonatal hepatitis. In that study, a GGT level $>300$ IU/L had a sensitivity of $39.7 \%$ and a GGT/AST ratio $>2$ showed a high possibility of biliary atresia. ${ }^{[15]}$ In our series, the GGT/AST ratio was significantly higher in those with biliary atresia (mean 2.9, SD 3.7) than in those with neonatal hepatitis (mean 1.1, SD 1.8) and other diagnoses (mean 2.4, SD 3.9; $p<0.05)$. AST, ALT and ALP levels were high in all groups, with AST significantly higher in those with neonatal hepatitis (Table 1). Even though the AST and ALT may only be marginally elevated in early cases of biliary atresia, these enzymes increase as progressive liver damage occurs, as seen in our patients who presented late. The causes of neonatal hepatitis in our series included sepsis, cytomegalovirus infection and idiopathic neonatal hepatitis. The total and conjugated bilirubin levels were high in all the groups and were not significantly different $(p>0.05)$ between the three groups.

\section{Conclusion}

Ultrasonography has poor sensitivity but good specificity in screening for biliary atresia; however, other investigations are necessary to confirm the diagnosis. Many patients with biliary atresia were referred too late for surgical intervention. Therefore, all babies with cholestatic jaundice must be referred early for specialist evaluation and exclusion of biliary atresia.

Acknowledgement. We thank Prof. G van Biljon of the Department of Paediatrics and Child Health, for advice on a scientific writing course by the Department of Research and Innovation Support, University of Pretoria.

\section{References}

1. Yang J, Ma D, Peng Y, Song L, Li C. Comparison of different diagnostic methods for differentiating biliary atresia from idiopathic neonatal hepatitis. Clin Imaging 2009;33(6):439-446. [http://dx.doi.org/10.1016/j. clinimag.2009.01.003]

2. Poddar U, Thapa B, Bhattacharya A, Rao K, Singh K. Neonatal cholestasis: Differentiation of biliary atresia from neonatal hepatitis in a developing country. Acta Paediatr 2009;98(8):1260-1264. [http://dx.doi.org/10.1111/ j.1651-2227.2009.01338.x]

3. Cartledge P, Kevlani N, Shapiro L, McClean P. An audit of a community protocol for identifying neonatal liver disease. Arch Dis Child 2012;97(2):166168. [http://dx.doi.org/10.1136/adc.2009.179812]

4. Moyer V, Freese DK, Whitington PF, et al. Guideline for the evaluation of cholestatic jaundice in infants: Recommendations of the North American Society for Pediatric Gastroenterology, Hepatology and Nutrition. J Pediatr Gastroenterol Nutr 2004;39(2):115-128. [http://dx.doi.org/10.1097/00005176200408000-00001]

5. Nwomeh B, Caniono D, Hogan M. Definitive exclusion of biliary atresia in infants with cholestatic jaundice: The role of percutaneous cholecystocholangiography. Pediatr Surg Int 2007;23(9):845-849. [http:dx.doi org/10.1007/s00383-007-1938-2]

6. Takamizawa S, Zaima A, Muraji T, Akasaka Y, Satoh S, Nishijima E. Can biliary atresia be diagnosed by ultrasonography alone? J Pediatr Surg 2007;42(12):2093-2096. [http://dx.doi.org/10.1016/j.jpedsurg.2007.08.032]

7. Park W, Choi S, Lee H, Kim S, Zeon S, Lee S. A new diagnostic approach to biliary atresia with emphasis on ultrasonographic triangular cord sign: Comparison of ultrasonography, hepatobiliary scintigraphy and liver needle biopsy in the evaluation of infantile cholestasis. J Pediatr Surg 1997;32(11);1555-1559. [http://dx.doi.org/10.1016/S0022-3468(97)90451$6]$

8. Hartley JL, Davenport M, Kelly DA. Biliary atresia. Lancet 2009;374(9702):17041713. [http://dx.doi.org/10.1016/S0140-6736(09)60946-6]

9. Dehghani S, Mahmood H, Imanieh M, Geramizadeh B. Comparison of different diagnostic methods in infants with cholestasis. World J Gastroenterol 2006;12(36):5893-5896

10. Deghady MAA, Abdel-Fattah M, Abdel-Kader M, Naguib M, Madina E, Abd El Gawad M. Diagnostic evaluation of cholestasis in infants and young children in Alexandria. Internet J Pediatr Neonatol 2005;6(1). http://www.ispub.com/ IJPN/6/1/8863 (accessed 26 February 2015).

11. Chardot C, Serinet M. Prognosis of biliary atresia: What can be further improved? J Pediatr 2006;148(4):432-434. [http://dx.doi.org/10.1016/j. jpeds.2006.01.049]

12. Davenport M, Puricelli V, Farrant P, et al. The outcome of the older ( $\geq 100$ days) infant with biliary atresia. J Pediatr Surg 2004;39(4):575-581. [http://dx.doi. org/10.1016/j.jpedsug.2003.12.014]

13. Tripepi G, Jager KJ, Dekker FW, Zoccali C. Statistical methods for the assessment of prognostic biomarkers (Part 1): Discrimination. Nephrol Dial Transplant 2010;25:1399-1401.

14. Elfaramawy A. Cholestasis in neonates and infants. Egypt J Med Hum Genet 2008;9(2):135-147.

15. Tang K, Huang Y, Lai C, et al. Gamma glutamyl transferase in the diagnosis of biliary atresia. Acta Paediatr Taiwan 2007;48(4):196-200. 